\title{
ANTIHYDROGEN FORMATION IN LOW ENERGY COLLISION OF POSITRON WITH ANTIPROTON
}

\author{
S. Binttacharyya \\ Gokhale Memorial Girls' College, Calcutta-700 020, India \\ (Reccived May 4, 1993; revised version March 16, 1994)
}

\begin{abstract}
In the present paper two-body radialive recombination rate for the production of antihydrogen (II) in a merged bean of slow positrons $\left(\mathrm{e}^{+}\right.$) and antiprotons $\left(\mathrm{p}^{-}\right)$is studied in the light of a two-sicp process, which consists of capture in an excited state of $\overline{I I}$ with subsequent decay to the ground state and emission of a photon. Computation is done using the field theory and the Coulomb gauge. Importauce of the two-step radiative recombination process relative to the well-known spontaneous photorecombination process, on the two-body radiative recombination rate for antiliydrogen formation, is discussed. The present result predicts higher contribution from the two-step radiative recombination process as compared to the spontaneous photorecombination process to the rate of cold antilydrogen formation with the relative collision energy below 0.01 Rydberg, near which experiments are being conducted. However, above 0.1 Rydberg the spontaneous photorecombination process dominates over the two-step radiative recombination process. The present result is valid, as well, for the formation of hydrogen atom due to collision between slow electron and proton.
\end{abstract}

PACS numbers: 31.10.+x, 34.50.+Fa, 34.70.+e

\section{Introduction}

The importance of antiproton as new form of space propulsion is gradually being realised. Few milligrams of antiprotons will heat tons of reaction fluid to high temperaturcs. The hot reaction fluid exhausted from a nozzle will produce high thrust at high exhaust velocity (100 to $350 \mathrm{~km} / \mathrm{s})$. For propulsion applications annihilation of antiprotons with protons producing jets of high energy pions is necessary. As cliarged antiprotons are difficult to store, it is desirable to store antiprotons in the form of neutral antihydrogen ice by adding positrons. Antihydrogen is also necessary to verify some fundamental properties of matter. The low energy antihydrogen can be used to measure the $2 S-2 p$ Lamb shift, the hydrogen-antihydrogen atomic interaction and for detection of gravitational effect on antimatter. 
Antihydrogen formation at LEAR (Low Energy Antiprotion Ring at CERN in Genewa) has been first considered by IIerr et al. [1], and subsequently, Gabrielse et al. [2] have discussed the possibility of antihydrogen formation by merging cold trapped plasmas of antiprotons and positrons. In the experiment [1] circulating antiproton beam of low divergence and momentum spread is merged with positron beam, in a straight section of a storage ring. After radiative recombination the antiprotons emerge from the cooling section of a storage ring tangentially (Fig. 1). In a merged beam the radiative recombination of antiproton and positron is pos-

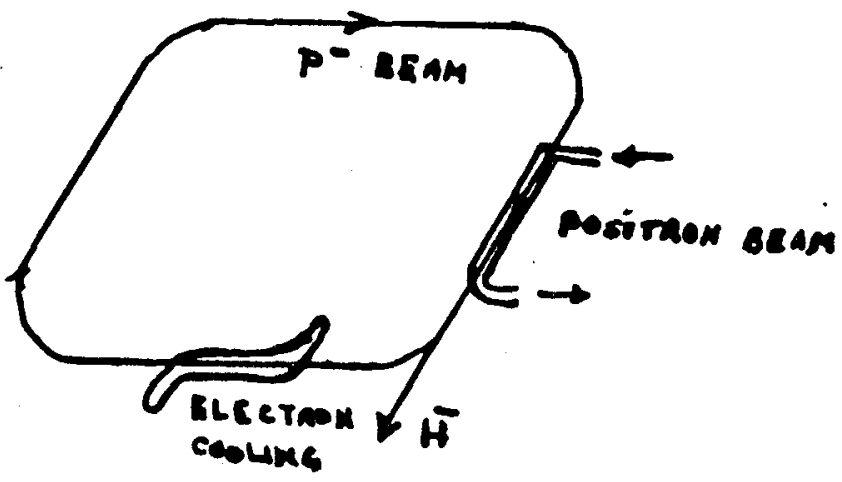

Fig. 1. Schematic setup for antilydrogen formation.

sible in a three-body (one antiproton and two positrons) as well as in two-body encounters. The radiative recombination reactions are shown in the equations below. Recombination is said to be complete when the antilydrogen is formed in the ground state.

$$
\begin{aligned}
& \mathrm{p}^{-}+\mathrm{e}^{+}+\mathrm{e}^{+} \rightarrow \stackrel{\overline{\mathrm{I}}(n l)}{\longrightarrow}+\mathrm{e}^{+} \\
& \mathrm{p}^{-}+\mathrm{e}^{+} \rightarrow \overline{\mathrm{I}}(l s)+h \nu, \\
& \mathrm{p}^{-}+\mathrm{e}^{+} \rightarrow \overline{\mathrm{I}}(n l) \rightarrow \overline{\mathrm{I}}(l s)+h \nu .
\end{aligned}
$$

The rates of the threc-body radiative recombination in (1) are obtained [3] from the principle of detailed balancing and the classical cross-section for collision ionization of Thomson [4] and Bohr [5] from an excited state $n$. The recombination rate is then multiplied by the transition probability to the ground state. The cross-section for two-body electron capture from continuum into the low lying Coulomb bound states of hydrogen-like systems will spontaneous photon emission was calculated $[6,7]$ in dipole approximations. This is a spontaneous photorecombination process (SPR) for hydrogen production. The process (2) gives SPR for antilyydrogen formation. SPR is also studied as a time reversed photoionization process [8]. The antilydrogen production rate by the three-body radiative recombination in (1) was, however, many orders of magnitude ligher [2] than the SPR rate in (2). 
In this paper we are interested in the two-body radiation recombination via the process (3) which provides an alternative path for the two-body radiative recombination. In case of process (3) a positron and an antiproton in the merged beam experiment experience Coulombic attraction to form antihydrogen in a higher orbit, which subsequently decays to the ground state with the emission of a photon. Momentum conservation in the final state is taken care of by the emitted photons. Eventually it is essential for the intermediate state to be an excited state from which radiative transition is possible. $\Lambda$ contribution from the process (3) towards radiative recombination of antiproton with positron is not yet computed. IIere we address ourselves to the problem of two-body two-step radiative recombination process (3) for the formation of antihydrogen. Cross-section for the two-step radiative recombination (TSRR) process is obtained by calculating the matrix element for the second order interaction consisting of the Coulomb attraction and dipole interaction. The state vectors and the interaction IIamiltonians arc taken in a ficld theoretic way and in the Coulomb gauge. Among all the excited intermediate states $2 p$-state lias the maximum probability for radiative decay to the ground state. We have computed TSRR cross-section with $2 p$ as the intermediate state and compared the result with that of SPR [7] for formation of hydrogen to the ground state. The comparative study reveals importance of TSRR in the two-body radiative recombination process to form antihydrogen (hydrogen) due to collision between cold antiproton (proton) and positron (electron).

\section{Mathcmatical formalism}

In the formalism of field theory, we make use of the Schrödinger wave function in momentum space and a Coulomb gauge. In the Coulomb gauge, interaction separates out into two parts: (1) the static Coulomb interaction, a part which is responsible for binding of electron (positron) to the nucleus to form bound state and (2) the interaction of the transverse electromagnetic field with the Dirac particles, a part largely responsible for ionization, recombination and optical transitions. Let us devclop below the field theoretic formalism to write the state vector for a system of two interacting particles, such as an clectron and a positron. The formalism will then be extended to write the state vectors for any number of interacting particles, including a photon [9].

In the Coulomb gauge, the field equation of quantum electrodynamics reads as

$$
\mathrm{i} \hbar \frac{\mathrm{d}}{\mathrm{d} \ell}|\psi\rangle=\left(H_{\mathrm{f}}+H_{\mathrm{C}}+I I\right)|\psi\rangle
$$

where the state vector of the field, $|\psi\rangle$, is taken as the direct product of the Dirac particle IIilbert space and photon IIilbert space. $I_{\mathrm{f}}$ is the IIamiltonian of the free Dirac particles and transverse photons. $H_{\mathrm{C}}$ is the Coulomb IIaniltonian and $H$ is the interaction IIamiltonian between Dirac particles and transverse electromagnetic field, and

$$
I_{\mathrm{C}}=\frac{e^{2}}{2} \int \frac{\rho(x) \rho\left(x^{\prime}\right)}{\left|x-x^{\prime}\right|} \mathrm{d}^{3} x \mathrm{~d}^{3} x^{\prime}
$$




$$
\begin{aligned}
& H=e \int \bar{\psi} \alpha \cdot \boldsymbol{A} \psi \mathrm{d}^{3} x+\frac{e^{2}}{2} \int \frac{\rho_{1}(x) \rho_{1}\left(x^{\prime}\right)}{\left|x-x^{\prime}\right|} \mathrm{d}^{3} x \mathrm{~d}^{3} x^{\prime} \\
& \quad+\frac{e^{2}}{2} \int \frac{\rho_{1}(x) \rho\left(x^{\prime}\right)}{\left|x-x^{\prime}\right|} \mathrm{d}^{3} x \mathrm{~d}^{3} x^{\prime}, \\
& \rho(x)=\bar{\psi}^{+}(x) \psi^{+}(x)+\bar{\psi}^{-}(x) \psi^{-}(x), \\
& \rho_{1}(x)=\bar{\psi}^{+}(x) \psi^{-}(x)+\bar{\psi}^{-}(x) \psi^{+}(x),
\end{aligned}
$$

where $\psi$ is the Dirac operator with $\psi^{+}(x)$ and $\psi^{-}(x)$ as their positive and negative frequency parts and $\boldsymbol{A}$ is the transverse electromagnetic field

$$
\psi(x)=\sum_{\sigma=1,2} a_{k} u_{\sigma}(k) \exp (\mathrm{i} k \cdot x)+\sum_{\sigma=1,2} b_{k} v_{\sigma}(-k) \exp (-\mathrm{i} k \cdot x) .
$$

Here $a_{k}$ and $b_{k}$ are the annihilation operators, for a free as well as bound particles, satisfying the anticommutation relations

$$
\left\{a_{k}^{\dagger}, a_{k}\right\}=0, \quad\left\{a_{k}^{\dagger}, I_{\mathrm{C}}\right\}=0 .
$$

Equation (4) is solved by the perturbation method. The unperturbed equation

$$
\mathrm{i} \hbar \frac{\mathrm{d}}{\mathrm{d} \ell}|\psi\rangle=\left(H_{f}+H_{\mathrm{C}}\right)|\psi\rangle
$$

includes the term $H_{\mathrm{C}}$, so that eigensolution $|\psi\rangle$ gives bound state as well as free particle distorted plane waves. In the present case of two particles, one electron and one positron, the state vector $|\psi\rangle$ is written as

$$
|\psi\rangle=\exp (-\mathrm{i} E l / h) \int g\left(k_{1}, k_{2}\right) a_{k_{1}}^{\dagger} b_{k_{2}}^{\dagger}|0\rangle \mathrm{d}^{3} k_{1} \mathrm{~d}^{3} k_{2} .
$$

That Eq. (10) is a solution of Eq. (9) is justified by showing that the corresponding wave function in configuration space

$$
\phi\left(x_{1}, x_{2}\right)=\left\langle 0\left|\psi\left(x_{1}\right) \psi^{\mathrm{c}}\left(x_{2}\right)\right| \psi\right\rangle
$$

is a solution of the equation

$$
\left(c p_{1} \alpha_{1}+\beta_{1} m c^{2}+c p_{2} \alpha_{2}+\beta_{2} m c^{2}-\frac{e^{2}}{r_{12}}\right) \phi\left(x_{1}, x_{2}\right)=E \phi\left(x_{1}, x_{2}\right) .
$$

As such, $g\left(k_{1}, k_{2}\right)$ is the Fourier transform in momentum space of the solution $\phi\left(x_{1}, x_{2}\right)$ of the electron-positron Eq. (12) with the Coulomb interaction. IIowever, in non-relativistic case, Eq. (12) becomes Pauli-Schrödinger equation with

$$
\rho(x)=\psi^{*}(x) \psi(x) \text {. }
$$

\section{Ficld-theoretic cross-section}

We use the above formalism to write the state vectors of the interacting systems. Positron-antiproton state vectors, in the initial state, and after recombination to the intermediate state, are written respectively as

$$
\left|\psi_{\mathrm{i}}\right\rangle=\exp \left(-\mathrm{i} E_{\mathrm{i}} l / h\right) \int g_{\mathrm{i}}\left(q_{1}, l_{1}\right) a_{q_{1}}^{\dagger} B_{l_{1}}^{\dagger}|0\rangle \mathrm{d}^{4} I_{1} \mathrm{~d}^{3} l_{1}
$$




$$
\left|\psi_{\mathrm{I}}\right\rangle=\exp \left(-\mathrm{i} E_{1} t / \hbar\right) \int g_{1}\left(q_{2}, l_{2}\right) a_{q_{2}}^{\dagger} B_{l_{2}}^{\dagger}|0\rangle \mathrm{d}^{3} q_{2} \mathrm{~d}^{4} l_{2},
$$

where $a_{q_{1}}$ and $B_{l_{1}}$ are the annihilation operators for positron and antiproton, respectively. $g_{\mathrm{i}}\left(q_{1}, l_{1}\right)$ and $g_{\mathrm{I}}\left(q_{2}, l_{2}\right)$ are the Fouricr transforms in momentum space of the free and the bound state solutions of the unperturbed equations

$$
\left(I_{0}+V\right) \phi_{\mathrm{i}}\left(x_{1}, x_{2}\right)=E_{\mathrm{i}} \phi_{\mathrm{i}}\left(x_{1}, x_{2}\right)
$$

and

$$
\left(H_{0}+V\right) \phi_{1}\left(x_{1}, x_{2}\right)=E_{1} \phi_{1}\left(x_{1}, x_{2}\right)
$$

respectively, with

$$
H_{0}=H_{\mathrm{e}+}+H_{\mathrm{p}^{-}}, \quad V=\int \frac{\rho(x) \sigma\left(x^{\prime}\right)}{\left|x-x^{\prime}\right|} \mathrm{d}^{3} x \mathrm{~d}^{3} x^{\prime} .
$$

$H_{\mathrm{e}^{+}}, H_{\mathrm{p}^{-}}$are the free particle IIamiltonians for the suffixed particles. $\rho(x)$ and $\sigma(x)$ are the charge densities for positron and antiproton, respectively. $V$ is the Coulomb interaction so that $\phi_{i}\left(x_{1}, x_{2}\right)$ and $\phi_{I}\left(x_{1}, x_{2}\right)$ contain respectively free particle distorted plane wave and positron-antiproton bound wave in an excited state.

The final state contains an antiproton-positron bound state and a photon. Let $C_{k}$ be the annililation operator for the photon with momentum $k$. The final state vector, with $|0\rangle$ as the vacuum state for particles and photon, is written as

$$
\left|\psi_{\mathrm{f}}\right\rangle=\exp \left(-\mathrm{i} E_{\mathrm{f}} t / \hbar\right) \int g \mathfrak{f}\left(\boldsymbol{q}_{3}, l_{3}\right) a_{q_{3}}^{\dagger} B_{l_{3}}^{\dagger} C^{\dagger}{ }_{k}|0\rangle \mathrm{d}^{3} l_{3} \mathrm{~d}^{3}{ }^{3} l_{3},
$$

where $g_{\mathrm{r}}\left(q_{3}, l_{3}\right)$ is the Fourier transform in momentum space of the unperturbed solution of the equation

$$
\left(H_{0}+I_{k}+V\right) \phi_{\mathrm{f}}\left(x_{1}, x_{2}\right)=E_{\mathrm{f}} \phi\left(x_{1}, x_{2}\right),
$$

where $H_{k}=\hbar \omega_{k} C_{k} C_{k}$ is the IIamiltonian for the emitted photon, $\phi_{\mathrm{f}}\left(x_{1}, x_{2}\right)$ is the wave function for positron and antiproton in $1 s$ bound state. Charge densities for positron and antiproton are respectively

$$
\rho(x)=e \phi^{*}(x) \phi(x)
$$

and

$$
\sigma(x)=-e \Theta^{*}(x) \Theta(x),
$$

where $\phi(x)$ and $\Theta(x)$, the respective field operators in the non-relativistic case, are written as

$$
\phi(x)=\sum_{r} \int \chi_{r} a_{s}^{\dagger} \exp (\mathrm{i} s \cdot x) \mathrm{d}^{3} s
$$

and

$$
\Theta(x)=\sum_{r^{\prime}} \int \lambda_{r^{\prime}} B_{s^{\prime}}^{\dagger} \exp \left(\mathrm{i} s^{\prime} \cdot x\right) \mathrm{d}^{3} s^{\prime},
$$

$\chi_{r}$ and $\lambda_{r^{\prime}}$ are the Pauli spinors for $\mathrm{e}^{+}$and $\mathrm{p}^{-}$, respectively.

The interaction IIamiltonian for Coulombic attraction between $\mathrm{e}^{-}$and $\mathrm{p}^{+}$is given by

$$
H_{1}=\int \frac{\rho(x) \sigma\left(x^{\prime}\right)}{\left|x-x^{\prime}\right|} \mathrm{d}^{3} x \mathrm{~d}^{3} x^{\prime} .
$$


The interaction between antiatom and the electromagnetic radiation field is given by [10]:

$$
\begin{aligned}
& H_{2}=\frac{e}{m c} p \cdot A(x)+\frac{e^{2}}{2 m c^{2}} A^{2}(x), \\
& H_{2}=H^{\prime}+H^{\prime \prime} .
\end{aligned}
$$

$\boldsymbol{p}$ is the momentum opcrator and $\boldsymbol{A}(x)$ is the electromagnetic field operator, which at a fixed time, is given by [10]:

$$
\boldsymbol{A}(x)=\sum_{k_{\sigma}^{\prime}}\left(\frac{2 \pi h c^{2}}{\Omega \omega_{k^{\prime}}}\right)^{1 / 2} u_{k_{\sigma}^{\prime}}\left[c_{k^{\prime}} \exp \left(\mathrm{i} k^{\prime} \cdot x\right)+c_{k^{\prime}}^{\dagger} \exp \left(-\mathrm{i} k^{\prime} \cdot x\right)\right]
$$

$u_{k_{\sigma}^{\prime}}$ is the polarization vector.

For emission of a photon by an excited atom, we consider only the first term in $H_{2}$ which is $I^{\prime} . S$ - matrix for the process is

$$
S=1+\left(H_{1}+I H_{2}\right)+H_{1} I_{1}+H_{2} H_{1}+\text { highicr order terms. }
$$

The radiative recombination (3) through the two-step process is obtained by taking the matrix element of $H_{2} H_{1}$ between initial and final states such that

$$
M_{\mathrm{f}}=\sum_{\mathrm{I}} \frac{\left\langle\psi_{\mathrm{f}}\left|I_{2}\right| \psi_{\mathrm{I}}\right\rangle\left\langle\psi_{\mathrm{I}}\left|I_{1}\right| \psi_{\mathrm{i}}\right\rangle}{\left(E_{\mathrm{i}}-E_{\mathrm{I}}+\mathrm{i} \eta\right)}
$$

$E_{\mathrm{i}}$ and $E_{\mathrm{I}}$ are the relative encrgies of the interacting systems in the initial and intermediate states, respectively, and the quantity $\eta$ is positive infinitesimal. After substituting from (21) and (22) in (25) and integrating over the coordinate space we get

$$
\begin{aligned}
H_{1}= & -e^{2} \int \frac{\delta^{3}\left(s_{1}-s_{2}+s_{1}^{\prime}-s_{2}^{\prime}\right)}{\left|s_{1}-s_{2}\right|} \\
& \times a_{s_{1}}^{\dagger} a_{s_{2}} B_{s_{1}^{\prime}}^{\dagger} B_{s_{2}^{\prime}} \chi_{r_{1}} \chi_{r_{2}} \lambda_{r_{1}^{\prime}} \lambda_{r_{2}^{\prime}} \mathrm{d}^{3} s_{1} \mathrm{~d}^{3} s_{2} \mathrm{~d}^{3} s_{1} \mathrm{~d}^{3} s_{2}^{\prime} .
\end{aligned}
$$

For a single photon emission the interaction term $I I^{\prime}$ of $H_{2}$ in (27) makes the first order contribution to the matrix element

$$
H^{\prime}=\frac{e}{m c} p \cdot A(x)
$$

\subsection{Probabilily for anlihydrogen formalion in the intermediale slate} and (31)

Matrix element of $I_{1}$ between $\left|\psi_{1}\right\rangle$ and $\left|\psi_{i}\right\rangle$ is obtained on using (14), (15)

$$
\begin{aligned}
M_{1} & =\left\langle\psi_{1}\left|I_{1}\right| \psi_{i}\right\rangle \\
& =\int g_{1}^{*}\left(q_{2} \cdot l_{2}\right) g_{i}\left(q_{1} \cdot l_{1}\right) \frac{\delta^{3}\left(s_{1}-s_{2}+s_{1}^{\prime}-s_{2}^{\prime}\right)}{\left|s_{1}-s_{2}\right|^{2}} \chi^{*}{ }_{r_{2}} \chi_{r_{1}} \lambda^{*}{ }_{r_{2}^{\prime}} \lambda_{r_{1}^{\prime}} \\
& \times\left\langle 0\left|a_{q_{2}} B_{l_{2}} a_{s_{1}}^{\dagger} a_{s_{2}} B_{s_{1}^{\prime}}^{\dagger} B_{s_{2}}^{\dagger} a_{q_{2}}^{\dagger} B_{l_{1}}^{\dagger}\right| 0\right\rangle \prod_{i=1,2}^{3} \mathrm{~d}^{3} q_{i} \mathrm{~d}^{3} l_{i} \mathrm{~d}^{3} s_{i} \mathrm{~d}^{4} s_{i}^{\prime} .
\end{aligned}
$$


Vacuum expectation value of the field operators gives product of Dirac $\delta$-functions. Integrating out the $\delta$-functions we get

$$
M_{1}=\int g_{1}^{*}\left(g_{2}, l_{2}\right) g_{i}\left(q_{1}, l_{1}\right) \frac{\delta^{3}\left(q_{2}-q_{1}+l_{2}-l_{1}\right)}{\left|q_{2}-q_{1}\right|^{2}} \chi_{q_{2}}^{*} \chi_{q_{1}} \lambda_{l_{2}}^{*} \lambda_{l_{1}} \prod_{i=1,2} \mathrm{~d}^{3} g_{i} \mathrm{~d}^{3} \boldsymbol{l}_{i} .
$$

Looking at Eqs. (16) and (17) the wave function in momentum space with associated Pauli spinor can be written as [9]:

$$
g_{\mathrm{i}}\left(q_{1}, l_{1}\right) \chi_{q_{1}} \lambda_{l_{1}}=\int \phi_{\mathrm{i}}\left(x_{1}, x_{2}\right) \exp \left(\mathrm{i} q_{1} \cdot x_{1}+\mathrm{i} l_{1} \cdot x_{2}\right) \mathrm{d}^{3} x_{1} \mathrm{~d}^{3} x_{2} .
$$

Changing the integration variables into centre of mass and relative coordinates and neglected the mass ratio between positron and antiproton we get

$$
g_{\mathrm{i}}\left(q_{1}, l_{1}\right)=\phi_{\mathrm{C}}\left(q_{1}\right) \lambda_{q_{1}}^{*} \lambda_{l_{1}}^{*} \delta^{3}\left(q_{1}+l_{1}-p_{\mathrm{c}}\right),
$$

where $p_{c}$ is the centre of mass momentum. $\phi_{\mathrm{C}}\left(\boldsymbol{q}_{1}\right)$ is the Coulomb distorted plane wave of the incident positron in momentum space. Similarly

$$
g_{1}\left(I_{3}, l_{2}\right)=\phi_{n l}\left(I_{2}\right) x_{q_{2}}^{*} \lambda_{l_{2}}^{*} \delta^{3}\left(I_{2}+l_{2}-Q_{1}\right),
$$

$Q_{1}$ is the centre of mass momentum of the intermediate system and $\phi_{n l}\left(g_{2}\right)$ is the $n l$-state bound positron wave function in momentum space. Substituting (36) and (37) in (34) we get after integration over $l_{1}$ and $l_{2}$

$$
M_{1}=-e^{2} \delta^{3}\left(p_{\mathrm{c}}-Q_{1}\right) \int \frac{\phi_{n 1}^{*}\left(q_{2}\right) \phi_{\mathrm{C}}\left(q_{1}\right)}{\left|q_{1}-q_{2}\right|^{2}} \mathrm{~d}^{3} \eta_{1} \mathrm{~d}^{3} \eta_{2}
$$

Using Beth integral we get

$$
M_{1}=-e^{2} \delta^{3}\left(p_{\mathrm{c}}-Q_{1}\right) \int \frac{\psi_{n l}(r) F_{\mathrm{C}}(r)}{|r|} \mathrm{d}^{3} r
$$

$F_{\mathrm{C}}(r)$ is the Coulomb distorted plane wave for incident positron and $\psi_{n l}(r)$ is the $n l$-state bound wave function of positron in antiliydrogen.

\subsection{Radialive decay of antihydrogen from nl-slate to 1 s ground state}

$H^{\prime}$ in (27) connects these states in the first order contribution to the radiative decay. The decay amplitude $M_{2}$ on using (32) and (28) becomes

$$
\begin{aligned}
M_{2} & =\left\langle\psi_{\mathrm{f}}\left|I_{2}\right| \psi_{\mathrm{I}}\right\rangle=\frac{e}{m c}\left\langle\psi_{\mathrm{f}}|\boldsymbol{p} \cdot \boldsymbol{A}(x)| \psi_{\mathrm{I}}\right\rangle \\
& =\frac{e}{m c} \sum_{k_{\sigma}^{\prime}}\left(\frac{2 \pi \hbar c}{\Omega \omega_{k^{\prime}}}\right)^{1 / 2} C_{k}\left\langle\psi_{\mathrm{f}}\left|p \cdot u_{k_{\sigma}^{\prime}} \exp \left(-\mathrm{i} k^{\prime} \cdot x\right)\right| \psi_{\mathrm{I}}\right\rangle
\end{aligned}
$$

Since the wave length of the emitted photon is larger than atomic dimension one 


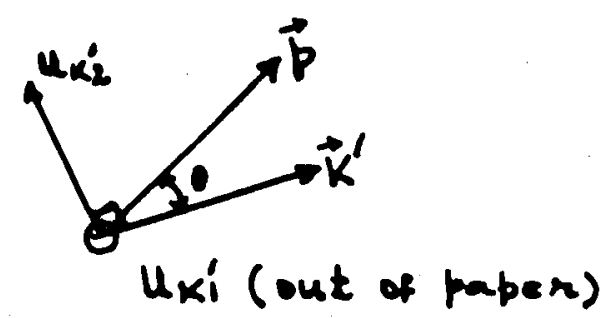

Fig. 2. Vector diagram showing the polarization and momentum directions.

can use dipole approximation. To sum over polarization we choose $\boldsymbol{u}_{k_{1}^{\prime}}$ and $\boldsymbol{u}_{k_{2}^{\prime}}$ as in Fig. 2 and obtain

$$
\begin{aligned}
& \sum_{\sigma=1,2}\left\langle\psi_{\mathrm{f}}\left|p \cdot u_{k^{\prime} \sigma} \exp (-\mathrm{i} k \cdot x)\right| \psi_{\mathrm{I}}\right\rangle=\left\langle\psi_{\mathrm{f}}\left|p \exp \left(-\mathrm{i} k_{k} \cdot x\right)\right| \psi_{\mathrm{I}}\right\rangle \sin \theta \\
& =\left\langle\psi_{\mathrm{f}}|p| \psi_{\mathrm{I}}\right\rangle \sin \theta \quad \text { [Dipole approximation] } \\
& =\left\langle\psi_{\mathrm{f}}\left|\frac{m}{\hbar} \frac{\mathrm{d} x}{\mathrm{~d} t}\right| \psi_{\mathrm{I}}\right\rangle \sin \theta=\left(\frac{-\mathrm{i} m}{\hbar}\right)\left\langle\psi_{\mathrm{f}}\left|x H_{\mathrm{op}}-H_{\mathrm{op}} x\right| \psi_{\mathrm{I}}\right\rangle \sin \theta \\
& =\left(\frac{-\mathrm{i} m}{\hbar}\right)\left(E_{\mathrm{I}}-E_{\mathrm{f}}\right)\left\langle\psi_{\mathrm{f}}|x| \psi_{\mathrm{I}}\right\rangle \sin \theta \\
& =\left(\frac{-\mathrm{i} m}{\hbar}\right)\left(\epsilon_{n l}-\epsilon_{1 s}\right)\left\langle\psi_{\mathrm{f}}|x| \psi_{\mathrm{I}}\right\rangle \sin \theta,
\end{aligned}
$$

where $H_{\mathrm{op}}=H_{0}+\mathrm{V}$ is the unperturbed IIamiltonian operator for positron bound to antiproton in the intermediate and the final state of the antihydrogen. Substituting (40), (15) and (19) in (39) and taking the vacuum expectation value for the product of field operators, and integrating over the momentum space

$$
M_{2}=\frac{-\mathrm{i} m}{\hbar} \bar{C}\left(\hbar \omega_{k}\right) \int \psi_{n l}(r) r \psi_{1 s}(r) \sin \theta \mathrm{d}^{3} r
$$

where

$$
\hbar \omega_{k}=\epsilon_{n l}-\epsilon_{1 s} \text { and } \bar{C}=\frac{e}{m c}\left(\frac{2 \pi \hbar c^{2}}{\omega_{k}}\right)^{1 / 2},
$$

$\epsilon_{n l}$ and $\epsilon_{1 s}$ are respectively the positron energy in $n l$ and $1 s$ bound states. The decay rate for radiative transition from $n l$ state to $1 s$ state is given by

$$
\tau_{n l \rightarrow 1 s}^{-1}=\int \frac{2 \pi}{\hbar} \delta\left(E_{\mathrm{i}}-E_{\mathfrak{f}}\right)\left|M_{2}\right|^{2} \frac{\mathrm{d}^{3} k}{(2 \pi)^{3}} .
$$

\subsection{Radialive recombination probability}

From (30) the radiative recombination probability $M_{\mathrm{f}}$ is given by

$$
\left|M_{\mathrm{f}}\right|=\left|M_{1}\right|\left|M_{2}\right| /\left|E_{\mathrm{i}}-E_{\mathrm{I}}+\mathrm{i} \eta\right| \text {. }
$$

The radiative recombination cross-section for the process becomes

$$
\sigma=\frac{2 \pi}{\hbar} \int \delta\left(E_{\mathrm{i}}-E_{\mathrm{f}}\right) \frac{m}{|\hbar p|} \frac{\mathrm{d}^{3} k}{(2 \pi)^{3}} \frac{\mathrm{d}^{3} p^{\prime}}{(2 \pi)^{3}}\left|M_{\mathrm{f}}\right|^{2} .
$$


$p$ and $\boldsymbol{p}^{\prime}$ are the relative momenta of the interacting systems before and after interaction. Using (13) and (42) in (11), the cross-section becomes

$$
\sigma=\tau_{n l \rightarrow 1 s}^{-1} \int \frac{m}{|h i p|} \frac{\mathrm{d}^{3} p^{\prime}}{(2 \pi)^{3}} \frac{\left|M_{1}\right|^{2}}{\left|E_{\mathrm{i}}-E_{\mathrm{i}}\right|^{2}} \text {. }
$$

Substituting (38) for $M_{1}$

$$
\sigma=\tau_{n l \rightarrow 1 s}^{-1} \int \frac{m}{|h p|} \frac{\mathrm{d}^{3} \eta^{\prime}}{(2 \pi)^{3}} \frac{e^{4} \delta^{3}\left(p_{\mathrm{c}}-Q_{\mathrm{I}}\right)}{\left|E_{\mathrm{i}}-E_{\mathrm{I}}\right|^{2}}\left|I_{n l}\right|^{2} .
$$

Integrating out the nomentum $\delta$-function

$$
\delta=\tau_{n l \rightarrow 1 s}^{-1} \frac{e^{4}}{(2 \pi)^{3}} \frac{m}{|h p|} \frac{\left|I_{n l}\right|^{2}}{\left|E_{\mathrm{i}}-E_{\mathrm{I}}\right|^{2}},
$$

where

$$
I_{n l}=\int \frac{\Psi_{n l}(r) F_{\mathrm{C}}(r)}{|r|} \mathrm{d}^{3} r
$$

\section{Results and discussions}

The cross-section for TSRR to the $2 p$ intermediate state which decays to the $1 s$ state with the emission of Lyman photon, is calculated. From (46)

$$
\sigma=\frac{\tau_{2 p-1 s}^{-1}}{\left|E_{\mathrm{i}}-E_{1}\right|} \frac{e^{4}}{(2 \pi)^{3}} \frac{m}{|\hbar p|}\left|\int \frac{\mid \psi_{2 p}(r) F_{\mathrm{C}}(r)}{|r|} \mathrm{d}^{3} r\right|^{2} .
$$

$\psi_{2 p}(r)$ is the $2 p$ orbital wave function of the antilydrogen. At low relative velocity the eflect of distortion on the plane wave of incident positron in the Coulomb field of the antiproton is obtained by taking

$$
F_{\mathrm{C}}(r)=(2 \pi|\xi|)^{1 / 2} \exp \left(\left.\right|_{\boldsymbol{p}} \cdot \boldsymbol{r}\right)
$$

where $f(\xi)=(2 \pi|\xi|)$ is the Sommerfeld factor [11] and

$$
\xi=-\frac{e^{2} m}{\hbar p}
$$

\section{Since}

$$
\psi_{2 p}(r)=N r \exp (-r / 2 a) \cos 0,
$$

the integral $I_{2 p}$ in (47) becomes

$$
I_{2 p}=\int \frac{\psi_{2 p}(r) F_{\mathrm{C}}(r)}{|r|} \mathrm{d}^{3} r=(2 \pi|\xi|)^{1 / 2}(-4 \pi \mathrm{i}) N F(p),
$$

where $a$ is the Bohr radius, the nomalization factor

$$
N=\left(\frac{2}{\pi a^{3}}\right)^{1 / 2} \frac{1}{8 a}
$$

and

$$
F(p)=\left[2 a^{2} p\left(p^{2}+\frac{1}{4 a^{2}}\right)^{2}\right]^{-1}
$$


The required radiative recombination cross-section (47) becomes

$$
\sigma=\frac{L}{E_{\mathrm{i}}} \frac{F^{2}(p)}{\left|E_{\mathrm{i}}-E_{\mathrm{I}}\right|^{2}}
$$

where

$$
\frac{L}{E_{\mathrm{i}}}=16 \pi^{2} N^{2} \frac{e^{4}}{(2 \pi)^{3}} \frac{1}{\tau_{2 p \rightarrow 1 s}} \frac{m}{|| i p \mid} \int(\xi) .
$$

The encrgy $E_{\mathrm{I}}$ in the intermediate stale is now given by

$$
E_{\mathrm{I}}=E_{\mathrm{i}}+c_{2 p}
$$

and

$$
\left|E_{\mathrm{i}}-E_{\mathrm{i}}\right|=\epsilon_{2 p} .
$$

The TSRR cross-section with $2 p$ as the intermediate state finally becomes

$$
\sigma=\frac{L}{E_{\mathrm{i}}} \frac{F(p)^{2}}{\epsilon_{2 p}^{2}} .
$$

Using absolute units

$$
E_{\mathrm{i}}=1.6 \times 10^{-12} \iota_{\mathrm{i}} \mathrm{crgs}, \quad \tau_{2 p \rightarrow 1 s}=1.6 \times 10^{-9} \mathrm{~s}
$$

and $t_{\mathrm{i}}$ the kinetic energy in $\mathrm{eV}$ of incident positron relative to antiproton, we compute the cross-section $\sigma$ (Table). The two special cases to see dependence of $\sigma$ on energy $E_{\mathrm{i}}$ are considered below.

\section{Case I}

$$
|p| \ll \frac{1}{2 a}, \quad \text { i.e. } \quad E_{\mathrm{i}} \ll \epsilon_{2 p} .
$$

Using this condition we get from (51)

$$
F(p)=\frac{8 a^{2}}{|p|}
$$

and the cross-section (52) becomes

$$
\sigma=\frac{32 a^{4} L h^{2}}{m \epsilon_{2 p}^{2}} \frac{1}{E_{i}^{2}} .
$$

When the relative collision encrgy $E_{\mathrm{i}}$ is low compared to $2 p$-state orbital energy $\epsilon_{2 p}$, the TSRR cross-section varies as $\mathbb{E}_{\mathrm{i}}^{-2}$.

\section{Case II}

$$
|p| \gg \frac{1}{2 a}, \quad \text { i.c. } \quad E_{\mathrm{i}} \gg \epsilon_{2 p}
$$

Condition (58) leads to

$$
F(p)=\left(2 a^{2} p^{5}\right)^{-1}
$$

and

$$
\sigma=\frac{L}{\epsilon_{2 p}^{2}} \frac{1}{4 a^{4}}\left(\frac{\hbar^{2}}{2 m}\right)^{5} \frac{1}{E_{\mathrm{i}}^{6}} .
$$

When the relative collision cnergy $E_{\mathrm{i}}$ is high compared to $\epsilon_{2 p}$, the TSRR cross-section varies as $E_{\mathrm{i}}^{-6}$. 
TABLE

Radiative recombination cross-section $\sigma$ for the formation of antiliydrogen in the ground state in unit of $10^{-20} \mathrm{~cm}^{2}$, due to collision between cold positron and antiproton. $E_{\mathrm{i}}$ is the encrgy in Rydberg of positron in antiproton rest frame. $\sigma_{\text {TSRR }}$ is the present result for the two-step radiative recombination cross-sections. $\sigma_{\mathrm{SPR}}$ is the result from Ref. [7] for spontancous photorecombination cross-sections.

\begin{tabular}{c|c|c|c|c|c|c}
\hline \hline$E_{\mathrm{i}}$ (in Rydberg) & $5^{-4}$ & $1^{-3}$ & $3^{-3}$ & $5^{-3}$ & $7^{-3}$ & $1^{-2}$ \\
\hline$\sigma_{\text {TSRR }}$ & $3.97^{3}$ & $1.003^{3}$ & $1.06^{2}$ & $3.68^{1}$ & $1.8^{1}$ & 8.4 \\
\hline$\sigma_{\text {SPR }}$ & $3^{1}$ & $1.8^{1}$ & $5^{1}$ & 3 & 1 & 1.8 \\
\hline$E_{\mathrm{i}}$ (in Rydberg) & $2^{-2}$ & $4^{-2}$ & $5^{-2}$ & $1^{-1}$ & $2.5^{-1}$ & $5^{-1}$ \\
\hline$\sigma_{\text {TSRR }}$ & 1.79 & $3.28^{-1}$ & $1.82^{-1}$ & $2.35^{-2}$ & $8.4^{-5}$ & $3.9^{-5}$ \\
\hline$\sigma_{\text {SPR }}$ & $9^{-1}$ & $4^{-1}$ & $3^{-1}$ & $1.6^{-1}$ & $6^{-2}$ & $2^{-2}$
\end{tabular}

Superscripts are powers of ten.

In a merged beam experiment the relative velocity of collision (56) should be kept well below $(2 \mathrm{ma})^{-1}$ to have a good antihydrogen formation rate. Table provides a basis for the comparative study of the contributions by TSRR and SPR mechanisms towards antiliydrogen formation. The SPR cross-section varies as $E_{\mathrm{i}}^{-1}$ [7], where as in the low energy limit the TSRR cross-section varies as $E_{\mathrm{i}}^{-2}$. Hence the TSRR cross-section is larger than the SPR cross-section in the low encrgy collision region. From Table, the antihydrogen formation cross sections for collision encrgy below 0.002 Rydberg are two orders of magnitude larger by TSRR mechanism as compared to that by the SPR mechanisms. Around 0.04 Rydberg, contributions from the both mechanisms arc almost of the same order of magnitude, with the SPR cross-sections remaining slightly higher than the TSRR cross-sections. With the increase in the collision encrgy above 0.1 Rydberg SPR cross-section dominates the radiative recombination process over TSRR cross-section. Near 0.25 Rydberg which is the $2 p$ statc binding energy, the TSRR cross-section is three orders of magnitude smaller than SPR cross-section.

\section{Conclusion}

An experiment for cooling of antiproton and positron to form $\overline{I I}$ in a merged beam technique is still in its carly stage [12]. The theoretical prediction on radiative recombination is available $[6,7]$ assuming the process to be SPR one. The present work brings out the importance of TSRR on the cold antihydrogen formation rate. The present result may be helpful for the ongoing experiment on antihydrogen formation. The result is also true for the TSRR cross-section for the formation of hydrogen atom with cold clectron and proton in a merged beam. This work provides an additional contribution to the formation rate over the SPR mechanism. 


\section{Acknowledgment}

The author likes to thank G. Gabrielse of IIarvard University, USA; and F.II.M. Faisal of Bielefeld University, Germany, for their helpful discussions on the paper. The work is supported by UGC, New Delhi, through the project number F 10-100/90 (RBB-II).

\section{R.corences}

[1] II. Ilerr, D. Mohl, A. Winnacker, in: Physics at LEAR wilh Low-Energy Cooled Antiprotons, Eds. U. Gastaldi, R. Klapisch, Plcuum Press, New York 1984, p. 659.

[2] G. Gabrielse, S.L. Rolster, L. Ilaarsma, W. Wells, Phys. Lelt. A 120, 38 (1988).

[3] N. D'Angels, Phys. Rev. 121, 505 (1960).

[4] J.J. Thomson, Philos. Mag. 23, 449 (1912).

[5] N. Bolır, Philos. Marg. 24, 10 (1913); 30, 581 (1915).

[6] R. Neuman, II. Poth, A. Winnacker, A. Wolf, Z. Phys. A 313, 253 (1983).

[7] M. Stobbe, Ann. Phys. 7, 661 (1930).

[8] M. Pajck, R. Scluch, Phys. Rcv. A 45, 7894 (1992).

[9] S. Bhattacliaryya, Indian J. Pure Appl. Phys. 22, 201 (1984).

[10] W. IIeitler, The Quantum Theory of Radiation, 3rd ecl., Oxford University Press, Oxford 1954, p. 175.

[11] H. Harris, C.M. Brown, Phys. Rcv. 105, 1656 (1957).

[12] R.L. Forward, in: Low Energy Anlimaller, Ed. D.B. Cline, World Scientific, Singapore 1986, p. 47. 